\section{Corrida em piscina funda promove manutenção da pressão arterial ao longo de cinco anos}

\section{Deep water running promotes maintenance of blood pressure over five years}

Thaís Reichert ${ }^{1}$

Ana Carolina Kanitz ${ }^{2}$

Rodrigo Sudatti Delevatti ${ }^{1,3}$

Luiz Fernando Martins Kruel ${ }^{1}$

\section{RESUMO}

O objetivo do presente estudo foi identificar o comportamento da pressão arterial sistólica, diastólica e média de repouso e máxima durante cinco anos de treinamento de corrida em piscina funda. A amostra foi composta por 39 indivíduos de meia idade, que foram divididos no grupo normotenso (43,6 6 7,38 anos, $120,00 \pm 11,54 / 80,00 \pm 10,00 \mathrm{mmHg}, \mathrm{n}=17$ ) e hipertenso $(59,27 \pm 7,00$ anos, $126,92 \pm 11,82 / 85,41 \pm 8,90 \mathrm{mmHg}, \mathrm{n}=22)$. Os sujeitos treinaram duas vezes por semana ( 45 minutos por sessão) de março a dezembro. O treinamento foi prescrito pela escala de esforço percebido de Borg (6-20) com o objetivo de promover ganhos cardiorrespiratórios. A avaliação cardiovascular foi realizada anualmente por um período de cinco anos, e os resultados de pressão arterial em repouso e máximo foram avaliados. Para análise dos dados, os testes de Shapiro-Wilk, Levene e ANOVA two-way com post-hoc de Bonferroni $(\alpha=0,05)$ foram utilizados. A pressão arterial sistólica, diastólica e média de repouso apresentaram uma manutenção ao longo dos cinco anos de treinamento em ambos os grupos, e os hipertensos tiveram os maiores valores. A pressão arterial sistólica máxima apresentou um decréscimo significativo do terceiro para o quarto ano em ambos os grupos, enquanto que a pressão diastólica e média máxima apresentaram uma manutenção ao longo do treinamento. Conclui-se que a prática de corrida em piscina funda promove uma manutenção dos valores da pressão arterial de repouso e máxima, em indivíduos normotensos e hipertensos. Este resultado é de grande relevância clínica, pois o avanço da idade tende a elevar os valores de pressão arterial, podendo resultar em doenças cardiovasculares.

\section{PALAVRAS-CHAVE}

Hipertensão; Treinamento; Exercícios aquáticos.

\begin{abstract}
The aim of this study was to identify the response of resting and maximal systolic, diastolic and mean blood pressure during five years of deep water running training. The sample was composed by 39 middle-aged individuals, which were divided in the normotensive (43.6 \pm 7.38 years, $120.00 \pm 11.54 / 80.00 \pm 10.00$ $\mathrm{mmHg}, n=17)$ and bypertensive group $(59.27 \pm 7.00$ years, $126.92 \pm 11.82 / 85.41 \pm 8.90 \mathrm{mmHg}$, $n=22)$. Subjects trained twice a week (45 minutes per session) from March to December. Training was prescribed by scale of perceived exertion of Borg (6-20) in order to promote cardiorespiratory gains. Cardiovascular assessment was performed annually for five years, and maximum and rest blood pressure were evaluated. For data analysis, Shapiro-Wilk, Levene, ANOVA two-way and Bonferroni's post-hoc $(\alpha=0.05)$ were used. The rest systolic, diastolic and mean blood pressure showed a maintenance during five years in both groups, and hypertensives had higher values. The maximal systolic blood pressure showed a significative decrease from the third to fourth year in both groups, whereas diastolic and mean blood pressure showed a maintenance during training. We conclude that deep water running promotes a maintenance of rest and maximal blood pressure values in normotensive and bypertensive individuals. This result is of great clinical relevance, because advancing age tends to increase blood pressure values, which can result in cardiovascular disease.
\end{abstract}

\section{KEYWORDS}

Hypertension; Training; Aquatic Exercises.
Rev Bras Ativ Fís Saúde p. 580-587 DOI

http://dx.doi.org/10.12820/rbafs.v.20n6p580

1 Universidade Federal do Rio Grande do Sul, Porto Alegre, Rio Grande do Sul, Brasil.

2 Universidade Federal de Uberlândia, Uberlândia, Minas Gerais, Brasil.

3 Faculdade Sogipa de Educação Física, Porto Alegre, Rio Grande do Sul, Brasil. 


\section{INTRODUÇÃO}

A hipertensão arterial sistêmica (HAS) é considerada um problema de saúde pública no Brasil, devido a sua magnitude, risco e dificuldades no seu controle ${ }^{1}$. A elevação dos níveis de pressão arterial (PA) é um importante fator de risco cardiovascular, cerebrovascular e renal, estando associada ao aumento da incidência de mortalidade da população ${ }^{2}$. Estima-se que cerca de 22,3 a $43,9 \%$ da população brasileira urbana adulta tenha o diagnóstico de $\mathrm{HAS}^{3}$, o que implica aos cofres públicos um gasto anual de US\$ 398,9 milhões (1,43\% dos gastos totais do SUS) para o tratamento da doença ${ }^{4}$.

$\mathrm{O}$ tratamento da HAS inclui medidas preventivas e terapêuticas, as quais podem ser de caráter farmacológico e não farmacológico. As medidas não farmacológicas estão relacionadas com as modificações do estilo de vida, como a inclusão de exercício físico ${ }^{1,2}$. Este é considerado uma ferramenta chave para a prevenção e controle da $\mathrm{HAS}^{5,6}$. Em relação ao modo de exercício, aqueles de caráter aeróbio têm demonstrado diminuírem significativamente a PA de indivíduos normotensos e hipertensos ${ }^{7}$. Porém, a grande maioria destes estudos $^{8-10}$ adotou como exercício a caminhada, corrida ou cicloergômetro no meio terrestre, não sendo muito explorado o fator meio de treinamento (aquático ou terrestre) no desfecho da PA.

Exercícios físicos realizados no meio aquático apresentam vantagens em relação aos exercícios realizados em meio terrestre, como o reduzido impacto $\operatorname{articular}^{11}$, uma menor ativação simpática, adrenérgica e a supressão do sistema renina angiotensina ${ }^{12}$, refletindo em menores valores de frequência cardíaca e PA durante a imersão. Essas características do meio aquático são de extrema valia para indivíduos obesos e idosos, público que apresenta maior prevalência e fator de risco para o desenvolvimento da HAS ${ }^{2,3}$. Apesar disso, pouco é conhecido acerca dos efeitos do treinamento em meio aquático sobre a $\mathrm{PA}^{13}$, devido à escassez de estudos nessa área. Uma recente revisão sistemática ${ }^{13}$ demonstrou que $80 \%$ dos 10 estudos abordados observou decréscimos significativos na PA após um treinamento em meio aquático. No entanto, a maioria destes estudos apresentou um período de duração de treinamento de no máximo 12 semanas. À vista disso, percebe-se que ainda faltam estudos que elucidem os efeitos crônicos do exercício em meio aquático sobre a PA, especialmente àqueles com um maior período de acompanhamento, visto que a PA aumenta progressivamente com o avanço da idade.

Dessa forma, o objetivo do presente estudo foi identificar o comportamento da pressão arterial sistólica (PAS), diastólica (PAD) e média (PAM) de repouso e máxima de indivíduos normotensos e hipertensos durante cinco anos de treinamento de corrida em piscina funda.

\section{MÉTODOS}

A amostra do estudo foi composta por 39 indivíduos de meia-idade divididos em dois grupos: normotensos ( $\mathrm{n}=17,7$ homens e 10 mulheres) e hipertensos ( $n=22,8$ homens e 14 mulheres). Considerou-se como hipertensos aqueles que apresentaram este diagnóstico médico no eletrocardiograma. Esta pesquisa foi aprovada pelo Comitê de Ética da Universidade Federal do Rio Grande do Sul (22332). 
Os sujeitos participavam de um projeto de extensão de corrida em piscina funda desenvolvido pela Escola de Educação Física da Universidade Federal do Rio Grande do Sul e possuíam tempo médio de prática de 2,84ะ 6,05 anos ao iniciarem o estudo. Caso o indivíduo apresentasse três faltas no mês, era excluído do projeto (com exceção para situações de doenças ou viagens). Ao realizar a matrícula no projeto, a cada ano, todos os participantes deveriam entregar um eletrocardiograma de esforço com o objetivo de verificar o seu estado de saúde. Este exame deveria conter as seguintes informações: idade, massa corporal, estatura, pressão arterial de repouso e máxima. A partir destes exames foram extraídos os dados para a realização deste estudo. Avaliou-se a PAS, PAD e PAM tanto de repouso como máxima. A PAM foi calculada por meio da seguinte fórmula: PAS + $\left(\mathrm{PAD}^{*} 2\right) / 3$.

A aferição da pressão arterial de repouso foi realizada após o indivíduo permanecer 15 minutos sentado (esfigmomanômetro Premium, 2000, China). Após isso, o teste máximo foi realizado de acordo com o protocolo de Bruce que consiste em um teste em esteira ergométrica, com inclinação inicial de $1 \%$ e velocidade de 2,73 Km.h ${ }^{-1}$ e incrementos de $2 \%$ na inclinação e 1,36 $\mathrm{Km} . \mathrm{h}^{-1}$ na velocidade a cada 3 minutos, até a exaustão do indivíduo. Os critérios para a interrupção do teste foram: atingir a FC máxima predita pela idade (220-idade) e indicar percepção de esforço maior que 17 (muito intenso Escala de Percepção de Esforço de Borg). Os sujeitos foram orientados a não ingerir estimulantes e bebidas alcoólicas, a não praticar exercício físico e a não utilizar o medicamento para controle da PA no dia do teste. Além disso, eles não deveriam realizar o teste em jejum.

O projeto de extensão de corrida em piscina funda do qual os indivíduos participavam iniciava no mês de março e encerrava em dezembro, sendo janeiro e fevereiro os meses de férias. O seu objetivo principal era a melhora cardiorrespiratória dos praticantes. Para isso, a periodização do treinamento era realizada a cada ano, sendo dividida em um macrociclo correspondente ao ano, mesociclos correspondente aos meses e microciclos correspondente às semanas. Março era considerado o mês de base (intensidade baixa, correspondente ao índice 13 da Escala de Percepção de Esforço de Borg (6-20)); abril e maio os meses de aquisição (intensidade moderada, correspondente aos índices 13 e 15 da Escala de Percepção de Esforço de Borg (6-20)); junho o primeiro pico da periodização (intensidade alta, correspondente aos índices 15 e 17 da Escala de Percepção de Esforço de Borg (6-20)) e julho um período regenerativo (intensidade baixa, correspondente ao índice $13 \mathrm{da}$ Escala de Percepção de Esforço de Borg (6-20)); no segundo semestre, os meses de agosto, setembro e outubro eram correspondentes aos meses de aquisição (intensidade moderada, correspondente aos índices 13 e 15 da Escala de Percepção de Esforço de Borg (6-20)), novembro o mês do segundo pico (intensidade alta, correspondente aos índices 15 e 17 da Escala de Percepção de Esforço de Borg (6-20)) e em dezembro eram realizadas atividades lúdicas e recreacionais (baixa intensidade, correspondente ao índice 13 da Escala de Percepção de Esforço de Borg (6-20)).

As aulas eram realizadas duas vezes por semana (segundas e quartas-feiras) em quatro horários $(16: 00 \mathrm{~h} ; 16: 50 \mathrm{~h} ; 17: 40 \mathrm{~h} ; 18: 30 \mathrm{~h})$ em uma piscina funda (1,95 metro de profundidade) com dimensões de 25 metros de comprimento, 16 metros de largura e temperatura da água controlada entre 30 a $32^{\circ} \mathrm{C}$. $\mathrm{Um}$ 
professor que permanecia fora da água, auxiliado por um monitor dentro da água, ambos estudantes de educação física, ministravam as aulas. As sessões eram compostas por cinco minutos de aquecimento, com caminhada em intensidade auto selecionada leve (índice 9 da Escala de Percepção de Esforço de Borg); 30 minutos de parte principal, destinada à corrida piscina funda concomitante à realização de exercícios para os principais grupos musculares de membros superiores (flexores/extensores horizontais de ombro, flexores/ extensores de ombro e cotovelo e abdutores e adutores de ombro); cinco minutos de exercícios de resistência muscular localizada para membros inferiores (adução/abdução de quadril) e abdominais, finalizando com cinco minutos de alongamento.

Para análise dos dados, utilizou-se estatística descritiva com os dados apresentados em média $\pm D P$. Foram utilizados os testes de Shapiro-Wilk e Levene para verificar a normalidade e homogeneidade dos dados, respectivamente. Para os dados não normais realizou-se uma transformação logarítmica com base 10. Para a comparação ao longo dos cinco anos de treinamento e entre os grupos utilizou-se ANOVA two-way (tempo e grupo) com post-hoc de Bonferroni. $\mathrm{O}$ índice de significância adotado foi $\alpha=0,05$ e foi utilizado o programa estatístico SPSS versão 20.0.

\section{RESULTADOS}

A caracterização da amostra pode ser visualizada na tabela 1.

\begin{tabular}{|c|c|c|}
\hline Variável & Hipertensos & Normotensos \\
\hline Idade (anos) & $59,27 \pm 7,00$ & $43,6 \pm 7,38$ \\
\hline Massa corporal (Kg) & $65,90 \pm 11,39$ & $69,65 \pm 11,00$ \\
\hline Estatura (m) & $1,75 \pm 0,11$ & $1,65 \pm 0,11$ \\
\hline Índice de massa corporal & $25,46 \pm 3,15$ & $25,08 \pm 7,26$ \\
\hline PAS repouso (mmHg) & $126,92 \pm 11,82$ & $120,00 \pm 11,54$ \\
\hline PAD repouso (mmHg) & $85,41 \pm 8,90$ & $80,00 \pm 10,00$ \\
\hline PAM repouso $(\mathrm{mmHg})$ & $184,44 \pm 18,68$ & $174,70 \pm 16,16$ \\
\hline FC (batimentos) & $81,00 \pm 15,34$ & $84,87 \pm 14,77$ \\
\hline $\begin{array}{l}\text { Medicamentos utilizados } \\
\text { (número de indivíduos que } \\
\text { utilizam) }\end{array}$ & $\begin{array}{c}\text { Inibidores da enzima de conversão } \\
\text { da angiotensina (ECA) (3), diuréticos } \\
\text { (3), bloqueadores de receptores beta- } \\
\text { adrenérgicos (2). }\end{array}$ & - \\
\hline $\begin{array}{l}\text { Comorbidades (número de } \\
\text { indivíduos) }\end{array}$ & $\begin{array}{c}\text { Sobrepeso (10), obesidade (1), } \\
\text { dislipidemia (1) }\end{array}$ & $\begin{array}{l}\text { Sobrepeso (6), obesidade } \\
\text { (4), dislipidemia (2) }\end{array}$ \\
\hline
\end{tabular}

Na figura 1, observam-se os valores da PAS, PAD e PAM de repouso e máxima durante os cinco anos de treinamento dos indivíduos normotensos e hipertensos. Em relação à situação de repouso, o grupo hipertenso apresentou os maiores valores em todas as variáveis (PAS: $p=0,015$; PAD: $p=0,019$; PAM: $\mathrm{p}=0,006)$ e não foi observada diferença significativa no fator tempo (PAS: $\mathrm{p}=0,857$; PAD: $\mathrm{p}=0,450$; PAM: $\mathrm{p}=0,623$ ) e na interação tempo* grupo (PAS: $\mathrm{p}=0,772$; PAD: $\mathrm{p}=0,497$; PAM: $\mathrm{p}=0,861)$. Em relação aos valores máximos de PA, não foram observadas diferenças significativas no fator grupo (PAS: $\mathrm{p}=0,431$; PAD: $\mathrm{p}=0,372$; PAM: $\mathrm{p}=0,066$ ), a PAS apresentou uma diminuição 
significativa no fator tempo do terceiro para o quarto ano $(\mathrm{p}=0,03)$, enquanto a PAD e PAM apresentaram uma manutenção ao longo do treinamento $(\mathrm{p}=0,567 ; \mathrm{p}=0,112$, respectivamente) e não foi observada interação significativa tempo*grupo (PAS: $\mathrm{p}=0,641$; PAD: $\mathrm{p}=0,454$; PAM: $\mathrm{p}=0,492$ ).
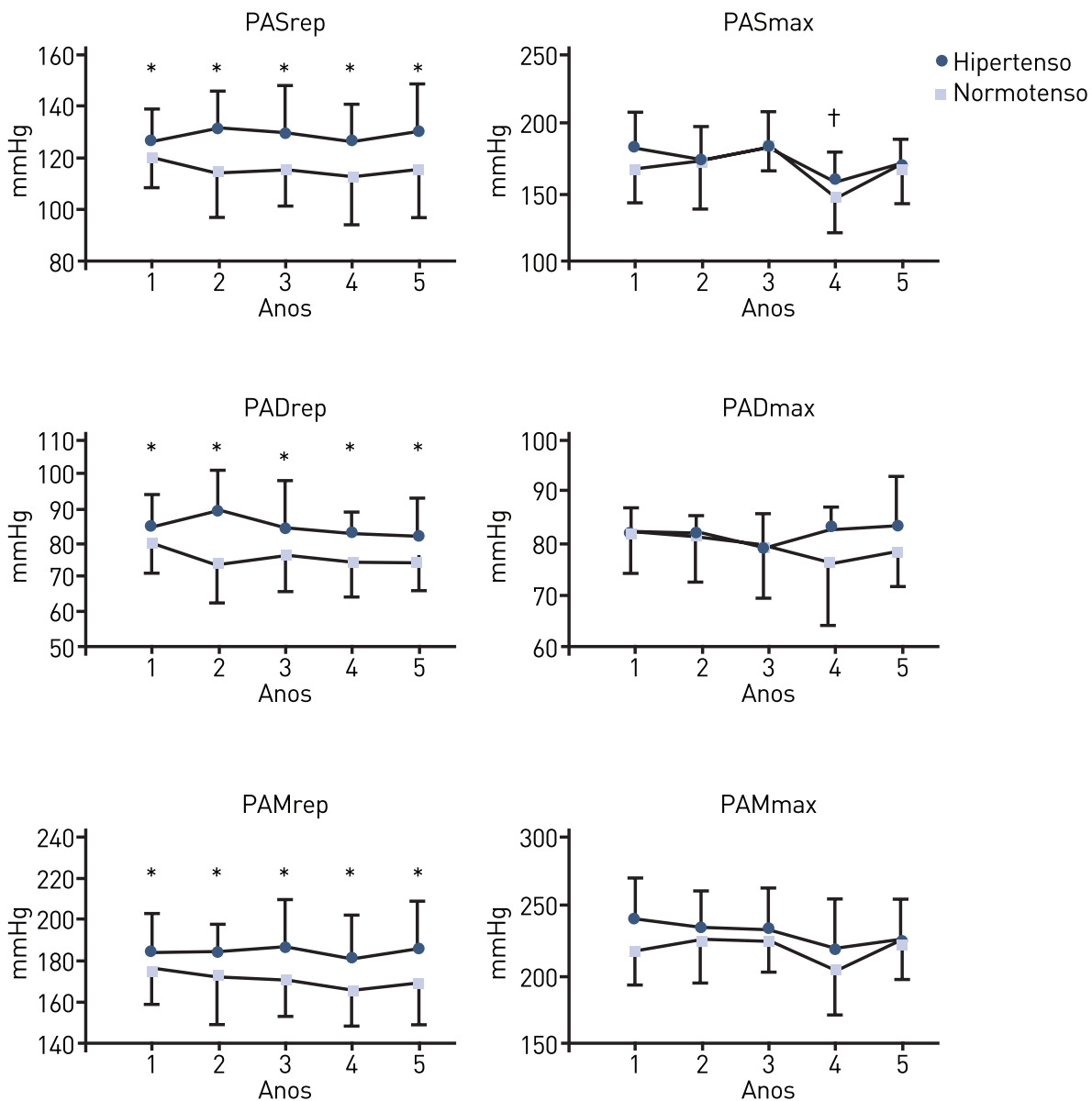

FIGURA 1 - Pressão arterial sistólica, diastólica e média de repouso e máxima do grupo hipertenso e normotenso durante os cinco anos de treinamento.

PAS: pressão arterial sistólica; PAD: pressão arterial diastólica; PAM: pressão arterial média; rep: repouso; max: máxima; HIPER: hipertensos; NORMO: normotensos. trepresenta diferença estatisticamente significativas entre os anos de treinamento para $p<0,05 .{ }^{*}$ representa diferença estatisticamente significativa entre os grupos para $p<0,05$.

\section{DISCUSSÃO}

O principal achado do presente estudo foi que a prática de corrida em piscina funda com frequência semanal de apenas duas sessões permitiu uma manutenção da pressão arterial de repouso e máxima de indivíduos de meia-idade normotensos e hipertensos ao longo de cinco anos.

No presente estudo, em cada ano de treinamento, os sujeitos possuíam dois meses de férias (janeiro e fevereiro) que eram precedidos por um mês de atividades lúdicas de baixa intensidade. Estes três meses de destreino podem ter sido responsáveis pela manutenção da PA encontrada. Ressalta-se, porém, que a manutenção observada é de grande relevância clínica, já que impediu o aumento da PA decorrente do processo de envelhecimento, visto que um período de apenas seis meses de inatividade física é capaz de elevar significa- 
tivamente a PA de repouso, deslocando os indivíduos de uma classificação de PA normal para hipertensão leve?.

Outros dois estudos da literatura avaliaram a PA de repouso após a prática de corrida em piscina funda ${ }^{14,15}$. Reichert ${ }^{14}$ verificou uma redução significativa da PAS e PAD de idosos após um treinamento de 28 semanas (grupo contínuo: $142,53 \pm 16,41$ e $88 \pm 3,76$ para $125,38 \pm 14,88$ e $77,18 \pm 7,75 \mathrm{mmHg}$; grupo intervalado: $133,58 \pm 15,43$ e $75,83 \pm 7,77$ para $123,16 \pm 17,39$ e $69,83 \pm 11,58$ mmHg). Já Delevatti et al. ${ }^{15}$ observaram uma manutenção da PAS e PAD de indivíduos com diabetes tipo 2 após 12 semanas de treinamento $(131,5 \pm 3,2$ e $75,2 \pm 1,8$ para $124,5 \pm 3,6$ e 71,9 $\pm 2,6 \mathrm{mmHg})$. Os diferentes níveis iniciais de PA dos estudos podem explicar os diferentes desfechos encontrados. Enquanto que os indivíduos do estudo de Reichert ${ }^{14}$ iniciaram o treinamento com níveis elevados de PA, os indivíduos do estudo de Delevatti et al. ${ }^{15}$, bem como os do presente estudo, possuíam valores normais de PA, conferindo-lhes uma menor amplitude para melhora ${ }^{1}$.

Em relação à PA máxima, no presente estudo não foi observada diferença significativa entre os indivíduos normotensos e hipertensos, demonstrando que a resposta da $\mathrm{PA}$ nos indivíduos hipertensos não apresentou um aumento excessivo durante a situação de exercício. Este resultado sugere que o treinamento de corrida em piscina funda permitiu o controle sobre a resposta da PA dos indivíduos hipertensos durante o exercício, fazendo com que o exercício físico, mesmo em situação máxima, não tenha incrementado de forma excessiva os níveis pressóricos dessa população.

Por fim, o resultado em relação à PAM, tanto em repouso quanto em exercício, também foi positivo. Essa variável representa a pressão média exercida pelo sangue na circulação pelas artérias ${ }^{16}$. Com o avanço da idade, ocorre um enrijecimento natural das artérias, fazendo com que haja uma tendência de aumento do valor da $\mathrm{PAM}^{16}$. Entretanto, o presente estudo demonstrou que a prática de corrida em piscina funda foi eficiente em limitar tal efeito deletério do envelhecimento, permitindo uma manutenção da PAM, contribuindo para reduzir a sobrecarga do miocárdio.

Os mecanismos de regulação da PA são multifatoriais. Em uma recente revisão, é discutido que o aumento da ativação simpática e a perda da sensibilidade baroreflexa causados pelo envelhecimento são os principais mecanismos de aumento da $\mathrm{PA}^{17}$. Nesse sentido, acreditamos que a manutenção dos níveis pressóricos encontrada deva estar relacionada com uma possível manutenção da atividade simpática e da sensibilidade baroreflexa por meio do exercício físico ${ }^{17}$. Além disso, o fato de o exercício físico ter sido realizado em meio aquático também pode ajudar a explicar os resultados. Quando o indivíduo está imerso, a pressão hidrostática aumenta o retorno venoso, fazendo com que mais sangue retorne ao coração e vasculatura intratorácica ${ }^{12}$. A hipervolemia central é responsável por aumentar o volume diastólico final e, consequentemente, aumentar também o volume sistólico. Com o aumento da distensibilidade atrial, estimulando receptores cardiopulmonares de baixa pressão, ocorre uma inibição no fluxo atividade nervosa simpática. A partir dessa inibição, ocorre uma redução na liberação de catecolaminas e de renina e, consequentemente, há uma redução da $\mathrm{PA}^{18}$. Desta forma, acreditamos que os efeitos somados da imersão e do exercício físico possibilitaram uma manutenção da atividade simpática e, consequentemente, da PA ao longo de cinco anos. 
Até onde se tem conhecimento, os resultados do presente estudo são inovadores na literatura, visto que não foi encontrado outro estudo que avaliasse a PA de indivíduos submetidos a um treinamento de tamanha duração. Os achados demonstraram que a prática de corrida em piscina funda permitiu uma importante prevenção primária, prevenindo que os indivíduos normotensos se tornassem hipertensos, já que há uma probabilidade que $90 \%$ dos indivíduos com PA normal até os 55 anos se tornem hipertensos ${ }^{19}$, e secundária, impedindo o aparecimento de complicações associadas à hipertensão como, por exemplo, o infarto do miocárdio e acidente vascular encefálico.

Possíveis limitações do presente estudo são a ausência de um grupo controle, que se tornou inviável devido à longa duração do período de intervenção e o não controle da idade em que as mulheres entraram na menopausa, que poderia vir a interferir nas variáveis avaliadas.

Conclui-se que a prática de corrida em piscina funda com mínima frequência semanal (duas sessões) é uma medida de baixo custo que promove uma manutenção dos valores de PAS, PAD e PAM, tanto em situação de repouso quanto em exercício, de indivíduos normotensos e hipertensos. Este resultado possui grande relevância clínica e contribui para o controle dos efeitos do processo de envelhecimento, que tendem a aumentar a PA.

\section{REFERÊNCIAS}

1. Sociedade Brasileira de Cardiologia / Sociedade Brasileira de Hipertensão / Sociedade Brasileira de Nefrologia. VI Diretrizes Brasileiras de Hipertensão. Arq Bras Cardiol 2010; 95(1 supl.1): 1-51.

2. Pescatello LS, Franklin BA, Fagard R, Farquhar WB, Kelley GA, Ray CA. American College of Sports Medicine Position Stand. Exercise and hypertension. Med Sci Sports Exerc. 2004; 36(3): 533-53.

3. Brasil. Ministério da Saúde. Secretaria de Atenção à Saúde. Departamento de Atenção Básica. Hipertensão arterial sistêmica para o Sistema Único de Saúde / Ministério da Saúde, Secretaria de Atenção à Saúde, Departamento de Atenção Básica. - Brasília : Ministério da Saúde, 2006. 58 p. - (Cadernos de Atenção Básica;(Série A. Normas e Manuais Técnicos)

4. Dib MW, Riera R, Ferraz MB. Estimated annual cost of arterial hypertension treatment in Brazil. Rev Panam Salud Publica. 2010; 27(2): 125-31.

5. Lenfant C, Chobanian AV, Jones DW, Roccella EJ. Seventh report of the Joint National Committee on the Prevention, Detection, Evaluation, and Treatment of High Blood Pressure (JNC 7): resetting the hypertension sails. Hypertension. 2003; 41(6): 1178-9.

6. Mancia G, Fagard R, Narkiewics K, Redon J, Zanchetti A, Bohm M, et al. 2013 ESH/ ESC Guidelines for the management of arterial hypertension: the Task Force for the management of arterial hypertension of the European Society of Hypertension (ESH) and of the European Society of Cardiology (ESC). J Hypertens. 2013; 31(7): 1281-357 .

7. Katzmarzyk PT, Leon AS, Wilmore JH, Skinner JS, Rao DC, Rankinen T, et al. Targeting the Metabolic Syndrome with Exercise: Evidence from the HERITAGE Family Study. Med Sci Sports Exerc. 2003; 35(10): 1703-9.

8. Cornelissen VA, Arnout J, Holvoet P, Fagard RH. Influence of exercise at lower and higher intensity on blood pressure and cardiovascular risk factors at older age. J Hypertens. 2009, 27(4): 753-62.

9. Sirbu E. The effects of moderate aerobic training on cardiorespiratory parameters in healthy elderly subjects. J Phys Educ Sport. 2012; 12(4): 560-3.

10. Miyai N, Arita M, Miyashita K, Morioka I, Shiraishi T, Nishio I, et al. Antihypertensive Effects of Aerobic Exercise in Middle-Aged Normotensive Men with Exaggerated Blood Pressure Response to Exercise. Hypertens Res. 2002; 25(4): 507-14. 
11. Kruel LFM. Alterações fisiológicas e biomecânicas em indivíduos praticando exercícios de hidroginástica dentro e fora d'água [tese de doutorado]. Santa Maria: Universidade Federal de Santa Maria; 2000.

12. Epstein M. Renal effects of head-out water immersion in humans: a 15-year update. Physiol Rev. 1992; 72(3): 563-621.

13. Santos N, Costa R, Kruel LFM. Efeitos de exercícios aeróbicos aquáticos sobre a pressão arterial em adultos hipertensos: revisão sistemática. Rev Bras Ativ Fis Saúde. 2014; 19(5): 548-58.

14. Reichert, T. Efeitos de dois modelos de aula de corrida em piscina funda nas respostas antropométricas, pressão arterial, aptidão física relacionada à saúde e qualidade de vida de idosos [monografia]. Porto Alegre: Universidade Federal do Rio Grande do Sul; 2014.

15. Delevatti RS, Kanitz AC, Alberton CL, Marson EC, Lisboa SC, Pinho CDF, et al. Glucose control can be similarly improved after aquatic or dry-land aerobic training in patients with type 2 diabetes: A randomized clinical trial. J Sci Med Sport. In press.

16. Wilmore JH, Costill DL, Kenney WL. Physiology of Sports and Exercise. United States: Human Kinetics, 2010.

17. La Rovere e Pinna. Beneficial Effects of Physical Activity on Baroreflex

18. Control in the Elderly. Ann Noninvasive Electrocardiol. 2014; 19(4) :303-10.

19. Coruzzi P, Novarini A, Musiari L, Rossi E, Borghetti A. Effects of 'central hypervolemia' by Water immersion on renin-aldosterone system and ACTH-cortisol axis in hemodialyzed patients. Nephron. 1984; 36(4):238-41.

20. Vasan RS, Beiser A, Seshadri S, Larson MG, Kannel WB, D'Agostino RB et al. Residual lifetime risk for developing hypertension in middle-aged women and men: the Framingham Heart Study. JAMA. 2002; 287:1003-10.

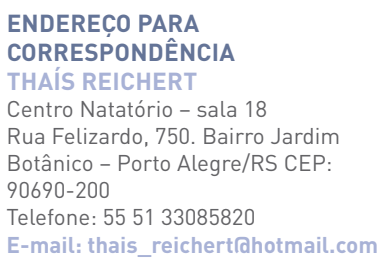

